\title{
Effect of Soy Isoflavones on Growth of Representative Bacterial Species from the Human Gut
}

\author{
Lucía Vázquez, Ana Belén Flórez, Lucía Guadamuro and Baltasar Mayo * \\ Departamento de Microbiología y Bioquímica, Instituto de Productos Lácteos de Asturias (IPLA), \\ Consejo Superior de Investigaciones Científicas (CSIC), Paseo Río Linares s/n, 33300 Villaviciosa, Spain; \\ lucia.vazquez@ipla.csic.es (L.V.); abflorez@ipla.csic.es (A.B.F.); luciagg@ipla.csic.es (L.G.) \\ * Correspondence: baltasar.mayo@ipla.csic.es; Tel.: +34-985-89-21-31
}

Received: 3 May 2017; Accepted: 4 July 2017; Published: 8 July 2017

\begin{abstract}
The present work aimed to assess the susceptibility of dominant and representative bacterial populations from the human gut to isoflavones and their metabolites. To do so, the minimum inhibitory concentration (MIC) of isoflavone glycosides, isoflavone aglycones, and equol to 37 bacterial strains was determined by broth microdilution. Additionally, for 10 representative strains, growth curves, growth rate $(\mu)$, and optical density $\left(\mathrm{OD}_{600 \mathrm{~nm}}\right)$ of the cultures at $24 \mathrm{~h}$ were also determined. MICs of daidzin, genistin, daidzein, and genistein were $>2048 \mu \mathrm{g} \mathrm{mL}^{-1}$ for all strains assayed, while that of equol ranged from $16 \mu \mathrm{g} \mathrm{mL}^{-1}$ for Bifidobacterium animalis subsp. animalis to $>2048 \mu \mathrm{g} \mathrm{mL}^{-1}$ for Enterobacteriaceae strains. Changes in growth curves, $\mu$, and final OD were observed among the species in the presence of all tested compounds. Genistein reduced $\mu$ of Bacteroides fragilis, Lactococcus lactis subsp. lactis, and Slackia equolifaciens, while both genistein and equol increased that of Lactobacillus rhamnosus and Faecalibacterium prausnitzii. Compared to controls, lower final OD in the presence of aglycones and equol were recorded for some strains but were higher for others. Altogether, the results suggest that isoflavone-derived compounds could modify numbers of key bacterial species in the gut, which might be associated with their beneficial properties.
\end{abstract}

Keywords: isoflavones; daidzein; genistein; equol; minimum inhibitory concentration; lactic acid bacteria; bifidobacteria; intestinal bacteria

\section{Introduction}

High intakes of soy-containing foods have been epidemiologically associated with less intense menopausal symptoms and a reduced risk of developing cardiovascular and neurodegenerative diseases and cancer [1]. Though soy contains many biologically active substances [2], its beneficial health effects have been attributed to its isoflavone content [3]. Isoflavones are phenolic compounds found naturally in plants (among which soy is one of the richest sources), the chemical structures of which resemble 17- $\beta$-oestradiol. They therefore have estrogenic effects [3]. In soy milk and unfermented soy foods, isoflavones mostly appear as isoflavone-glycoside conjugates (daidzin, genistin, glycitin) - the bioavailability and estrogenic activity of which are low [4]. To be absorbed and reach full activity, isoflavone aglycones (daidzein, genistein, glycitein) need to be released from the corresponding glycosides [5]. This is accomplished by cellular $\beta$-glucosidases and $\beta$-glucosidases from components of the gut microbiota [6]. Isoflavone aglycones can be metabolized further by cellular enzymes, plus others from components of the gut microbiota [7], to produce more active compounds (such as equol from daidzein) or inactive metabolites [8].

Some of the beneficial health effects attributed to isoflavones could come about via the stimulatory or inhibitory modulation of gut microbial populations. However, the effects of isoflavones on gut microbiota have been little examined [9-12]. Increases in the number of bifidobacteria have been 
recorded in some studies [10,12], and population sizes within Clostridium clusters have been reported to increase in equol producers $[10,13]$. However, reductions in bifidobacteria and populations of Enterobacteriaceae have been observed in other studies [11]. Such contradictory results may ultimately depend on the baseline size and composition of the bacterial communities in the gut, which can vary widely between subjects [14]. Finally, like many other polyphenols [15], isoflavones and some of their metabolites have been shown to possess a certain antimicrobial activity against bacterial pathogens [16-18]. It is thus conceivable that they might directly or indirectly alter the numbers or relative proportions of pivotal bacterial communities for maintaining a healthy microbial balance in the gut.

The present work aimed to examine the possible inhibitory effect of the most common soy isoflavone glycosides (daidzin and genistin), their derived aglycones (daidzein and genistein), and equol, against 37 bacterial strains, including lactic acid bacteria, bifidobacteria, and strains of other dominant and representative bacterial groups in the human gut.

\section{Materials and Methods}

\subsection{Bacterial Strains, Growth Media, and Culture Conditions}

Of the 37 strains used in this study, 25 were type strains of lactic acid bacteria (LAB) and bifidobacterial species obtained from the Laboratory of Microbiology collection in the Belgian Coordinated Collections of Microorganisms (BCCM/LMG) (Ghent University, Ghent, Belgium), 7 were strains (of which 6 were type strains) of species from human intestines obtained from the Deutsche Sammlung von Mikroorganismen und Zellkulturen (DSMZ) (Leibniz Institute, Braunschweig, Germany), and 5 strains of intestinal species were from our own laboratory collection (Table 1). Strains were considered representative of functional bacterial groups within the human gut; they also represent those most commonly used as probiotics. Lactococci were grown on M17 agar (Oxoid, Basingstoke, UK) supplemented with $1 \%$ glucose (VWR International, Radnor, PA, USA) at $32{ }^{\circ} \mathrm{C}$ for $48 \mathrm{~h}$ under aerobic conditions. Streptococcus thermophilus was cultured on M17 agar (Oxoid) supplemented with $1 \%$ lactose (VWR International) at $37^{\circ} \mathrm{C}$ for $48 \mathrm{~h}$, under anaerobic conditions. Heterofermentative lactobacilli were recovered on de Man, Rogosa, and Sharpe (MRS) (Merck, Darmstad, Germany) agar plates and incubated for $48 \mathrm{~h}$ at $32{ }^{\circ} \mathrm{C}$ or $37{ }^{\circ} \mathrm{C}$ and under aerobic or anaerobic conditions, depending on the species. Homofermentative lactobacilli and bifidobacteria were grown on MRS agar supplemented with $0.25 \%$ L-cysteine (Merck) and incubated at $37{ }^{\circ} \mathrm{C}$ for $48 \mathrm{~h}$ under anaerobic conditions. Intestinal anaerobic strains (Bacteroides spp., Blautia coccoides Faecalibacterium prausnitzii, Ruminococcus obeum, and Slackia spp.) were streaked on Gifu anaerobic medium (GAM) (Nissui, Tokyo, Japan). All strains of these species were incubated at $37^{\circ} \mathrm{C}$ for $48 \mathrm{~h}$ under anaerobic conditions. Finally, strains of Escherichia coli, Klebsiella pneumoniae, Pseudomonas aeruginosa, and Serratia marcescens were grown on brain heart infusion (BHI; Oxoid) agar at $37^{\circ} \mathrm{C}$ for $24 \mathrm{~h}$ under aerobic conditions.

\subsection{Determination of Minimum Inhibitory Concentration}

The minimum inhibitory concentration (MIC) of the majority of isoflavone glycosides in soy (daidzin and genistin), their respective aglycones (daidzein and genistein), and the isoflavone metabolite equol (all from LC Laboratories, Woburn, MA, USA) were determined using a broth microdilution test, following standard procedures for aerobic [19] and anaerobic bacteria [20] with minor modifications. Briefly, individual colonies from the above plates were suspended in $5 \mathrm{~mL}$ of a sterile $0.9 \% \mathrm{NaCl}$ solution (VWR International) to a McFarland turbidity of 1 . The inoculated saline solution was then diluted 1:1000 in the test medium corresponding to the different species (see Table 1) to obtain an approximate final concentration of $3 \times 10^{5} \mathrm{cfu} \mathrm{mL}^{-1}$. Aliquots $(100 \mu \mathrm{L})$ of the diluted cell suspensions were poured into microplate wells with $50 \mu \mathrm{L}$ of two-fold increasing concentrations of the test compounds, ranging from 0.12 to $2048 \mu \mathrm{g} \mathrm{mL}^{-1}$ (the limit of their solubility). MICs were 
established by visual inspection as the lowest concentration at which no visible growth was observed. All MIC assays were performed in duplicate. Where discrepancies between analyses were observed, a third assay was performed and the mode reported.

Table 1. Bacterial strains, assay conditions for the minimum inhibitory concentration (MIC), and MIC results of equol to the intestinal species and strains under study.

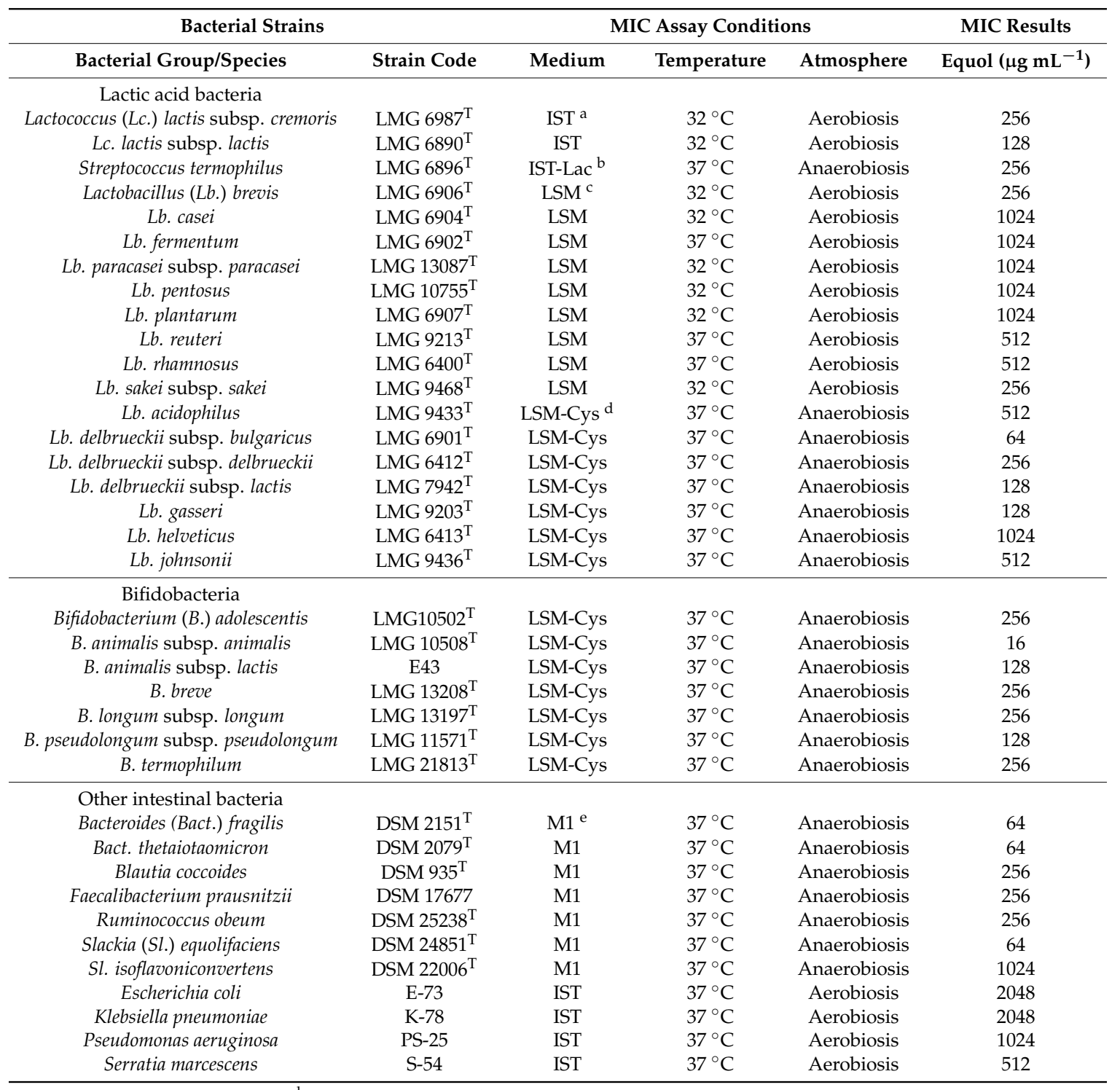

${ }^{\text {a }}$ IST, IsoSensitest (Oxoid); ${ }^{\mathrm{b}}$ IST-Lac (IST + $1 \%$ lactose); ${ }^{\mathrm{c}}$ LSM, Lactic acid bacterium susceptibility test medium $\left(90 \%\right.$ IST $+10 \%$ de Man, Rogosa and Sharpe (MRS)) $;{ }^{d}$ LSM-Cys (LSM + 0.03\% cysteine) $;{ }^{\text {e }}$ M1 (90\% IST + 10\% Gifu Anaerobic Medium (GAM) $+0.25 \%$ cysteine). MICs were assayed in duplicate or triplicate; when discrepancies were found, the mode was reported.

\subsection{Effect of Isoflavone Aglycones and Equol on Bacterial Growth}

The growth of bacteria in the presence of daidzein, genistein, and equol was monitored spectrophotometrically, measuring the optical density (OD) throughout culturing. Colonies were collected, suspended in $10 \mathrm{~mL}$ of an appropriate liquid medium, and incubated for $24 \mathrm{~h}$ under species-specific conditions as stated above. These cultures were then used to inoculate appropriate fresh media (at 1\%) supplemented in independent tubes with daidzein, genistein, or equol (all at $32 \mu \mathrm{g} \mathrm{mL}{ }^{-1}$ ). Cultures to which no phenolic compounds were added were used as controls. Growth was monitored by measuring the OD at $600 \mathrm{~nm}$ using the culture medium as a blank. All growth experiments were 
performed in triplicate; mean results are reported. The bacterial growth rate $(\mu)$ was calculated using the formula $\mu=\operatorname{Ln}\left(N_{2} / N_{1}\right) / t_{2}-t_{1}$, where $N_{1}$ was the OD at time $1\left(t_{1}\right)$ and $N_{2}$ was the OD at $t_{2}$. The interval $t_{1}-t_{2}$ was selected within the logarithmic growth phase of the different species and strains.

\subsection{Statistical Analysis}

Statistical analysis of the data was performed using the 3.2.5. version of the free $\mathrm{R}$ software (The R Foundation, Boston, MA, USA). Normality of the data was checked by the Shapiro-Wilk test. Mean differences between control cultures and cultures with isoflavones were assessed using the Student's $t$-test.

\section{Results and Discussion}

All strains grew at the maximum concentration of isoflavone glycosides (daidzin and genistin) and isoflavone aglycones (daidzein and genistein) used (MICs $>2048 \mu \mathrm{g} \mathrm{mL}^{-1}$ ). In contrast, susceptibility to equol ranged widely, from $16 \mu \mathrm{g} \mathrm{mL}^{-1}$ to $2048 \mu \mathrm{g} \mathrm{mL} \mathrm{m}^{-1}$ (Table 1). The strain most susceptible

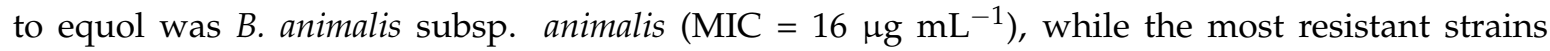
belonged to the Gram-negative species E. coli and K. pneumoniae ( $\mathrm{MIC}=2048 \mu \mathrm{g} \mathrm{mL}^{-1}$ ). Eight strains, among which five species of lactobacilli, Slackia isoflavoniconvertens, and P. aeruginosa were found, showed an MIC of $1024 \mu \mathrm{g} \mathrm{mL} \mathrm{m}^{-1}$. The tested strains of Lactobacillus delbrueckii subsp. bulgaricus, Bacteroides fragilis, Bacteroides thetaiotaomicron, and Slackia equolifaciens showed moderate susceptibility to equol (MIC $=64 \mu \mathrm{g} \mathrm{mL}^{-1}$ for all).

Overall, these results agree well with those reported in the literature, in which isoflavones lacking prenyl and hydroxyl groups at certain positions of the isoflavone ring structure-such as daidzin, genistin, daidzein and genistein—have shown no major antimicrobial activity [17]. Other studies describing isoflavones to have low antibacterial activity against Gram-negative bacteria have also been reported [16-18]. However, all these works had the aim of assessing isoflavones and their faecal-derived metabolites as potential antibacterial agents for counteracting the rise of antibiotic resistance among pathogens; this is why pathogenic species have been analysed so far [17]. In this work, a majority of strains under analysis were shown not to be inhibited by the tested compounds at concentrations higher than those reached at a physiological level $\left(\sim 200 \mu \mathrm{g} \mathrm{mL} \mathrm{m}^{-1}\right.$ of intestinal content under usual treatment regimens; [11]). However, due to the large microbial complexity and diversity within the human gut [14,21], the response to isoflavones and their metabolites of members of bacterial groups others than those analysed in this study might be different.

To determine whether isoflavones and equol could affect bacterial growth despite their high MIC values, the growth curves of 10 strains belonging to representative groups were investigated under specific culture conditions (see Section 2.1 and Table 1). As MICs of the isoflavone glycosides and isoflavone aglycones resulted identical, the former compounds were not tested in this assay. Since complete inhibition was not intended, the compounds to be assayed were added at a concentration below their MIC values $\left(32 \mu \mathrm{g} \mathrm{mL}^{-1}\right)$. Controls were prepared in which no phenolic compound was provided. Cultures were sampled hourly for the first $8 \mathrm{~h}$ of incubation and also after $24 \mathrm{~h}$ (at which time the maximum population size was attained). As expected, the growth kinetics recorded varied widely between bacterial groups (Figure 1). Except for slow-growing species (Sl. equolifaciens, Sl. flavoniconvertens, Faecalibacterium prausnitzii), standard deviation between assays was rather low for a microbial test, ranging from 0.03 to 0.23 . Sl. equolifaciens did not appreciably grow during the first $8 \mathrm{~h}$ of incubation in any of the cultures. Broadly speaking, growth of the majority of the strains during these first $8 \mathrm{~h}$ (up to the beginning of the stationary phase in most cases) was very similar in the presence or absence of the test compounds, suggesting them to have no effect. Such was the case for Lactobacillus gasseri, Lactobacillus plantarum, Bifidobacterium longum, E. coli, and S. marcescens (Figure 1A-C,E,F, respectively). Growth curves similar to those of L. gasseri and L. plantarum were also obtained for Lactobacillus rhamnosus (data not shown). In contrast, cultures of F. prausnitzii, Lactococcus lactis subsp. lactis, and Bact. fragilis were inhibited by equol and even strongly 
by genistein (Figure 1D,H,G, respectively). E. coli and S. marcescens grew better in the presence of isoflavones and equol than in the control cultures, although the difference was statistically significant for S. marcescens only (Figure 1F).
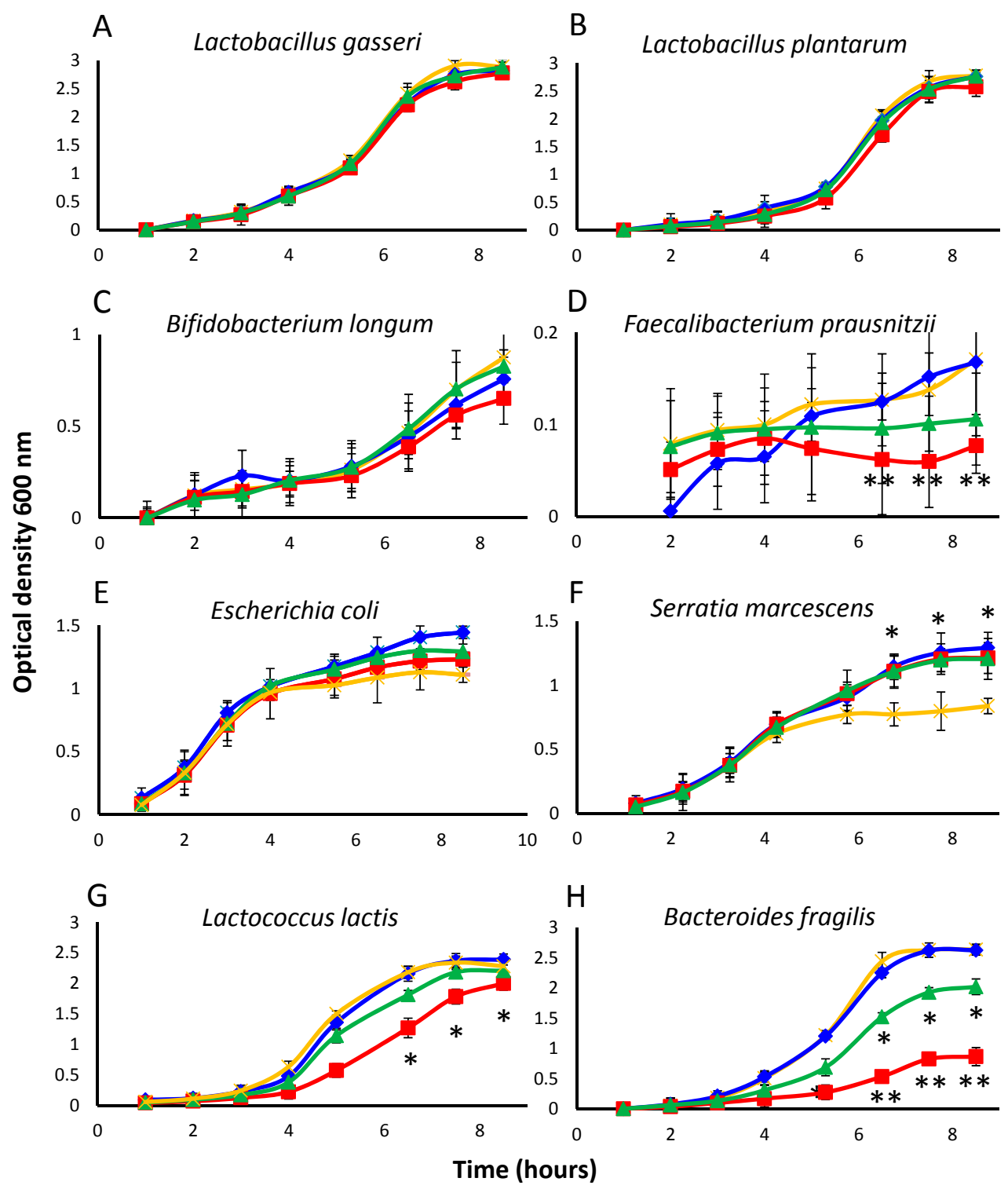

Figure 1. Growth curves of representative strains $(\mathbf{A}-\mathbf{H})$ as an average of the optical density (OD) measures of triplicate cultures in the presence of the soy isoflavone aglycones daidzein (in blue) and genistein (in red), and the daidzein-derived metabolite equol (in green) (all at $32 \mu \mathrm{g} \mathrm{mL}^{-1}$ ), as compared to a control without additives (in orange). Note that OD scale is different for different species. Mean values were compared by the Student's $t$-test. Vertical bars show standard deviations (SD). Statistical significance: ${ }^{*} p \leq 0.05,{ }^{* *} p \leq 0.01$.

Deconjugation of isoflavone glycosides leads to the release of free glucose [6], which could then be used as a fuel. However, degradation of aglycones by certain species and their use as an energy source cannot be discarded. Indeed, beyond the conversion of daidzein into equol and genistein into 5-hydroxyequol, the catabolic profiling of soy aglycones and their derived metabolites by (intestinal) bacteria has scarcely been addressed [22,23].

As compared to the controls, daidzein causes small increases or decreases in the growth rate $(\mu)$ depending on the species (Table 2). $\mu$ also decreased moderately in some strains when either genistein 
or equol was present in the culture medium, but increases were scored for some others. In accordance with the shape of their growth curves, the decrease in $\mu$ was particularly high for Bact. fragilis, L. lactis subsp. lactis, and Sl. equolifaciens. The enhanced growth rate of Lb. rhamnosus and S. marcescens in the presence of genistein and equol (Table 2) strongly suggests that somehow these species can degrade and use these compounds as an energy source. The catabolism of isoflavone glycosides, aglycones, and equol by strains of these species is currently underway. A particular case was F. prausnitzii. Equol and genistein inhibited growth of this species during the first $8 \mathrm{~h}$ of culture, but they both increased its $\mu$ value (calculated for this strain between 20 and $24 \mathrm{~h}$ ). To examine the effects of aglycones and equol on the maximum optical density (OD) attained by cultures, this parameter was evaluated for the 10 selected strains at $24 \mathrm{~h}$ (Figure 2). Compared to the controls and reinforcing the observed changes in the $\mu$, the presence of isoflavone aglycones or equol led to a lower final OD for some strains but higher for others. Of these changes, statistical significance was only found for the inhibition of Lb. gasseri by genistein, Bact. fragilis by both genistein and equol, and Sl. equolifaciens by all tested compounds.

Table 2. Growth rate of selected bacterial strains in cultures supplemented with daidzein, genistein, or equol at a final concentration of $32 \mu \mathrm{g} \mathrm{mL} \mathrm{m}^{-1}$ as compared to that in control cultures without isoflavone phenolics.

\begin{tabular}{|c|c|c|c|}
\hline Strain/Culture Conditions & Growth Rate $^{a}(\mu) h^{-1}$ & Species/Culture Conditions & Growth Rate $(\mu) h^{-1}$ \\
\hline Lb. gasseri LMG $9203^{\mathrm{b}}$ & & Bact. fragilis DSM $2151^{\mathrm{d}}$ & \\
\hline Control & 0.747 & Control & 0.681 \\
\hline Daidzein & 0.739 & Daidzein & 0.701 \\
\hline Genistein & 0.738 & Genistein & 0.153 \\
\hline Equol & 0.738 & Equol & 0.591 \\
\hline Lb. plantarum LMG $6907^{\text {b }}$ & & E. coli $\mathrm{E}-73^{\mathrm{e}}$ & \\
\hline Control & 0.791 & Control & 0.772 \\
\hline Daidzein & 0.755 & Daidzein & 0.775 \\
\hline Genistein & 0.776 & Genistein & 0.820 \\
\hline Equol & 0.722 & Equol & 0.787 \\
\hline Lb. rhamnosus LMG $6400^{\mathrm{b}}$ & & S. marcescens S-54 ${ }^{\mathrm{e}}$ & \\
\hline Control & 0.867 & Control & 0.808 \\
\hline Daidzein & 0.704 & Daidzein & 0.792 \\
\hline Genistein & 0.942 & Genistein & 0.874 \\
\hline Equol & 0.962 & Equol & 0.838 \\
\hline L. lactis subsp. lactis LMG $6890^{c}$ & & Sl. equolifaciens DSM $24851^{\mathrm{T}}$ & \\
\hline Control & 0.965 & Control & 0.238 \\
\hline Daidzein & 0.736 & Daidzein & 0.174 \\
\hline Genistein & 0.595 & Genistein & 0.121 \\
\hline Equol & 0.799 & Equol & 0.153 \\
\hline B. longum subsp. longum LMG $13197^{\text {b }}$ & & F. prausnitzii DSM 17677 & \\
\hline Control & 0.581 & Control & 0.187 \\
\hline Daidzein & 0.472 & Daidzein & 0.181 \\
\hline Genistein & 0.516 & Genistein & 0.232 \\
\hline Equol & 0.581 & Equol & 0.227 \\
\hline
\end{tabular}

${ }^{a}$ The specific growth rate $(\mu)$ under the culture conditions was calculated as $\mu=\operatorname{Ln}\left(N_{2} / N_{1}\right) / t_{2}-t_{1}$, where $N_{1}$ was the OD at $t_{1}$ and $N_{2}$ was the OD at $t_{2}$. To calculate $\mu$, a representative $t_{1}-t_{2}$ interval within the logarithmic growth phase of the cultures was selected. ${ }^{b}$ de Man Rogosa and Sharpe (MRS) broth supplemented with $0.25 \%$ cysteine. ${ }^{\mathrm{c}}$ M17 broth supplemented with 1\% glucose. ${ }^{\mathrm{d}}$ Gifu Anaerobic Medium (GAM) broth supplemented with $0.5 \%$ arginine. ${ }^{\mathrm{e}}$ Luria-Bertani (LB) broth.

The inhibitory activity of genistein against pathogens such as Staphylococcus aureus has been repeatedly reported $[18,24]$. As anticipated above, though the chemical structure of daidzein and genistein are very similar (except for the absence of an $\mathrm{OH}$ group in daidzein at position 5) [25], genistein inhibits DNA topoisomerease IV while daidzein does not [17], perhaps explaining its stronger antimicrobial action. It was surprising that the growth of Sl. equolifaciens was severely inhibited by all the test compounds; this and Sl. isoflavoniconvertens were the only equol-producing organisms among the tested bacteria. The equol used in this study was a racemic mixture of $R$ - and $S$-enantiomers, while only the latter is produced endogenously in the 
gut [8]. Therefore, as for some physiological effects [26], the antimicrobial action of the native equol might differ from that reported here. Moreover, soy isoflavones are metabolized into a vast array of chemically-related phenolic compounds $[27,28]$ such as dihydrodaidzein, dihydrogenistein, tetrahydrodaidzein, $O$-desmethylangolensin (O-DMA), 5-hydroxyequol, and others [7,8,22], whose antimicrobial behaviour was not tested in this study. In addition, other phenolics, such as 4-ethylcatechol, 3-phenylpropionic acid, 3-hydroxyphenylacetic acid, and 4-hydroxy-5-phenylvaleric acid, have also been recorded to increase their faecal concentrations after isoflavone consumption [22]. Intermediate or end-product metabolites might have a range of biological properties, including antimicrobial activity. In fact, an antimicrobial effect of phenylacetic and phenylpropionic acids has already been reported, particularly against Gram-negative intestinal pathogens [29]. To provide a complete picture of how these compounds affect communities of gut bacteria, the antimicrobial properties of more isoflavone-derived phenolic compounds against representative gut bacteria should be examined. Besides, the use of culture-independent molecular methods to assess the quantification of bacterial growth (such as real-time quantitative PCR) could bring about more accurate results than those obtained by the culturing approach used in this work.

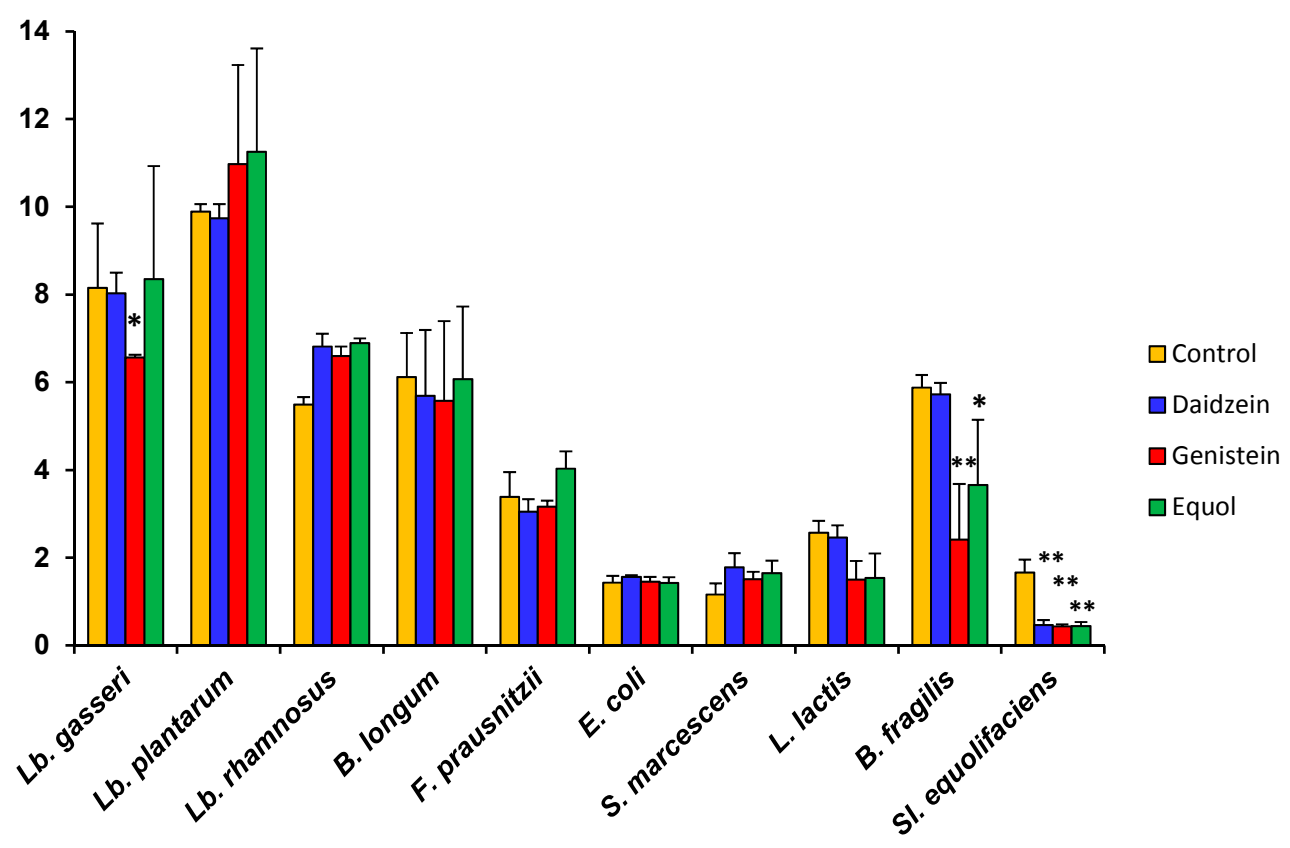

Figure 2. Final optical density (OD) at $600 \mathrm{~nm}$ after $24 \mathrm{~h}$ incubation of ten bacterial strains in the presence of $32 \mu \mathrm{g} \mathrm{mL}^{-1}$ of either daidzein, genistein, or equol, as compared to a control without phenolics. Standard deviation (SD) is indicated by vertical bars. Mean values were compared by the Student's $t$-test. Statistical significance: ${ }^{*} p \leq 0.05,{ }^{* *} p \leq 0.01$.

\section{Conclusions}

In conclusion, soy isoflavones and their metabolites are thought to have a range of beneficial health effects, which might be exerted through the modulation of bacterial populations in the human gut $[1,3,30]$. However, except for a few pathogens, studies examining the effects of these phenolic compounds on bacterial growth and metabolism have yet to be reported. To our knowledge, this is the first paper to report the resistance/susceptibility profiles of members of the commensal and beneficial bacterial communities of the human gut to isoflavones. The related parameters MIC, growth rate, and final growth estimate the competitiveness and fitness of bacteria in the presence of the compounds under study. Since isoflavone aglycones and equol can modify one or more of the variables examined, it might be concluded that when consumed either in food or in supplements, they may modify the total numbers and/or their relative proportions of specific bacterial communities in the gut. 
These modulatory effects on the intestinal bacterial populations might be associated with the beneficial properties attributed to soy consumption.

Acknowledgments: The study was partially supported by projects from the Spanish Ministry of Economy and Competitiveness (AGL-2014-57820-R) and Asturias Principality (GRUPIN14-137). L.G. and L.V. were supported by research contracts of the FPI Program from MINECO (BES-2012-062502 and BES-2015-072285, respectively). The skilful technical assistance of Paula Fernández is greatly acknowledged.

Author Contributions: L.V. and L.G. performed most of the experiments and contributed to the discussion of the results. A.B.F. performed experiments and critically review the manuscript. B.M. provided material and human resources, drafted and reviewed the manuscript.

Conflicts of Interest: The authors declare no conflict of interest.

\section{References}

1. Messina, M. Soy and health update: Evaluation of the clinical and epidemiologic literature. Nutrients 2016, 8, 754. [CrossRef] [PubMed]

2. Kang, J.; Badger, T.M.; Ronis, M.J.; Wu, X. Non-isoflavone phytochemicals in soy and their health effects. J. Agric. Food Chem. 2010, 58, 8119-8133. [CrossRef] [PubMed]

3. Pilšáková, L.; Riečanský, I.; Jagla, F. The physiological actions of isoflavone phytoestrogens. Physiol. Res. 2010, 59, 651-664. [PubMed]

4. De Cremoux, P.; This, P.; Leclercq, G.; Jacquot, Y. Controversies concerning the use of phytoestrogens in menopause management: Bioavailability and metabolism. Maturitas 2010, 65, 334-339. [CrossRef] [PubMed]

5. Islam, M.A.; Bekele, R.; Vanden Berg, J.H.; Kuswanti, Y.; Thapa, O.; Soltani, S.; van Leeuwen, F.X.; Rietjens, I.M.; Murk, A.J. Deconjugation of soy isoflavone glucuronides needed for estrogenic activity. Toxicol. In Vitro 2015, 29, 706-715. [CrossRef] [PubMed]

6. Landete, J.M.; Arqués, J.; Medina, M.; Gaya, P.; de Las Rivas, B.; Muñoz, R. Bioactivation of phytoestrogens: Intestinal bacteria and health. Crit. Rev. Food Sci. Nutr. 2016, 56, 1826-1843. [CrossRef] [PubMed]

7. Kim, M.; Han, J. Isoflavone metabolism by human intestinal bacteria. Planta Med. 2016, 81, S1-S381.

8. Franke, A.A.; Lai, J.F.; Halm, B.M. Absortion, distribution, metabolism, and excretion of isoflavonoids after soy intake. Arch. Biochem. Biophys. 2014, 59, 24-28. [CrossRef] [PubMed]

9. Clavel, T.; Fallani, M.; Lepage, P.; Levenez, F.; Mathey, J.; Rochet, V.; Sérézat, M.; Sutren, M.; Henderson, G.; Bennetau-Pelissero, C.; et al. Isoflavones and functional foods alter the dominant intestinal microbiota in postmenopausal women. J. Nutr. 2005, 135, 2786-2792. [PubMed]

10. Bolca, S.; Possemiers, S.; Herregat, A.; Huybrechts, I.; Heyerick, A.; De Vriese, S.; Verbruggen, M.; Depypere, H.; De Keukeleire, D.; Bracke, M.; et al. Microbial and dietary factors are associated with the equol producer phenotype in healthy postmenopausal women. J. Nutr. 2007, 137, 2242-2246. [PubMed]

11. Guadamuro, L.; Delgado, S.; Redruello, B.; Flórez, A.B.; Suárez, A.; Martínez-Camblor, P.; Mayo, B. Equol status and changes in faecal microbiota in menopausal women receiving long-term treatment for menopause symptoms with a soy-isoflavone concentrate. Front. Microbiol. 2015, 6, 777. [CrossRef] [PubMed]

12. Nakatsu, C.H.; Arsmstrong, A.; Cavijo, A.P.; Martin, B.R.; Barnes, S.; Weaver, C.M. Fecal bacterial community changes associated with isoflavone metabolites in postmenopausal women after soy bar consumption. PLoS ONE 2014, 9, e108924. [CrossRef] [PubMed]

13. Possemiers, S.; Bolca, S.; Eeckhaut, E.; Depypere, H.; Verstraete, W. Metabolism of isoflavones, lignans and prenylflavonoids by intestinal bacteria: Producer phenotyping and relation with intestinal community. FEMS Microbiol. Ecol. 2007, 61, 372-383. [CrossRef] [PubMed]

14. Lozupone, C.A.; Stombaugh, J.I.; Gordon, J.I.; Jansson, J.K.; Knight, R. Diversity, stability and resilience of the human gut microbiota. Nature 2012, 489, 220-230. [CrossRef] [PubMed]

15. Engels, C.; Schieber, A.; Gänzle, M.G. Inhibitory spectra and modes of antimicrobial action of gallotannins from Mango kernels (Mangifera indica L.). Appl. Environ. Microbiol. 2011, 77, 2215-2223. [CrossRef] [PubMed]

16. Hummelova, J.; Rondevaldova, J.; Balstikova, A.; Lapcik, O.; Kokoska, L. The relationship between structure in vitro antibacterial activity of selected isoflavones and their metabolites with special focus on antistaphylococcal effect of demethyltexatin. Lett. Appl. Microbiol. 2014, 60, 242-247. [CrossRef] [PubMed]

17. Mukne, A.P.; Viswanathan, V.; Phadatare, A.G. Structure pre-requisites for isoflavones as effective antibacterial agents. Pharmacogn. Rev. 2011, 5, 13-18. [CrossRef] [PubMed] 
18. Verdrengh, M.; Collins, L.V.; Bergin, P.; Tarkowski, A. Phytoestrogen genistein as an anti-staphylococcal agent. Microbes. Infect. 2004, 6, 86-92. [CrossRef] [PubMed]

19. CLSI (Clinical and Laboratory Standards Institute). Methods for Dilution Antimicrobial Susceptibility Tests for Bacteria That Grow Aerobically. Standard M07-A10, 10th ed.; CLSI: Wayne, PA, USA, 2015.

20. CLSI (Clinical and Laboratory Standards Institute). Methods for Antimicrobial Susceptibility Testing of Anaerobic Bacteria. Standard M11-A8, 8th ed.; CLSI: Wayne, PA, USA, 2012.

21. Sankar, S.A.; Lagier, J.C.; Pontarotti, P.; Raoult, D.; Fournier, P.E. The human gut microbiome, a taxonomic conundrum. Syst. Appl. Microbiol. 2015, 38, 276-286. [CrossRef] [PubMed]

22. Guadamuro, L.; Jiménez-Girón, A.M.; Delgado, S.; Flórez, A.B.; Suárez, A.; Martín-Álvarez, P.J.; Bartolomé, B.; Moreno-Arribas, M.V.; Mayo, B. Profiling of phenolic metabolites in feces from menopausal women after long-term isoflavone supplementation. J. Agric. Food. Chem. 2016, 64, 210-216. [CrossRef] [PubMed]

23. Schwen, R.J.; Nguyen, L.; Jackson, R.L. Elucidation of the metabolic pathway of S-equol in rat, monkey and man. Food Chem. Toxicol. 2012, 50, 2074-2083. [CrossRef] [PubMed]

24. Morán, A.; Gutiérrez, S.; Martínez-Blanco, H.; Ferrero, M.A.; Monteagudo-Mera, A.; Rodríguez-Aparicio, L.B. Non-toxic plant metabolites regulate Staphylococcus viability and biofilm formation: A natural therapeutic strategy useful in the treatment and prevention of skin infections. Biofouling 2014, 30, 1175-1182. [CrossRef] [PubMed]

25. Del Rio, D.; Rodriguez-Mateos, A.; Spencer, J.P.; Tognolini, M.; Borges, G.; Crozier, A. Dietary (poly)phenolics in human health: Structures, bioavailability, and evidence of protective effects against chronic diseases. Antiox. Redox Signal. 2013, 18, 1818-1892. [CrossRef] [PubMed]

26. Jackson, R.L.; Greiwe, J.S.; Schwen, R.J. Emerging evidence of the health benefits of S-equol, an estrogen receptor $\beta$ agonist. Nutr. Rev. 2011, 69, 432-448. [CrossRef] [PubMed]

27. Coldham, N.G.; Darby, C.; Hows, M.; King, L.J.; Zhang, A.Q.; Sauer, M.J. Comparative metabolism of genistin by human and rat gut microflora: Detection and identification of the end-products of metabolism. Xenobiotica 2002, 32, 45-62. [CrossRef] [PubMed]

28. Setchell, K.D.; Brown, N.M.; Zhao, X.; Lindley, S.L.; Heubi, J.E.; King, E.C.; Messina, M.J. Soy isoflavone phase II metabolism differs between rodents and humans: Implications for the effect on breast cancer risk. Am. J. Clin. Nutr. 2011, 94, 1284-1294. [CrossRef] [PubMed]

29. Lee, H.C.; Jenner, A.M.; Low, C.S.; Lee, Y.K. Effect of tea phenolics and their aromatic fecal bacterial metabolites on intestinal microbiota. Res. Microbiol. 2006, 157, 876-884. [CrossRef] [PubMed]

30. Selma, M.V.; Espín, J.C.; Tomás-Barberán, F.A. Interaction between phenolics and gut microbiota: Role in human health. J. Agric. Food Chem. 2009, 57, 6485-6501. [CrossRef] [PubMed] 